\title{
Yo-Yo Intermittent Recovery Level 2 Test Performance and Leg Muscle Growth in a Six-month Period among Pubescent Soccer Players at Different Stages of Maturity
}

\author{
Kentaro Chuman ${ }^{1,2}$, Yoshihiro Hoshikawa ${ }^{3}$, \\ Tomomi Iida $^{3}$ and Takahiko Nishijima ${ }^{4}$ \\ ${ }^{1}$ Doctoral Program in Physical Education, Health and Sport Sciences, University of Tsukuba, \\ 1-1-1 Tennodai, Tsukuba-shi, Ibaraki 305-8574 Japan \\ ${ }^{2}$ YAMAHA FOOTBALL CLUB CO., LTD., 2500 Shingai, Iwata-shi, Shizuoka 438-0025 Japan \\ ${ }^{3}$ Sports Photonics Laboratory, HAMAMATSU PHOTONICS K.K., \\ 2150-1 Iwai, Iwata-shi, Shizuoka 438-0016 Japan \\ ${ }^{4}$ Institute of Health and Sport Science, University of Tsukuba, \\ 1-1-1 Tennodai, Tsukuba-shi, Ibaraki 305-8574 Japan \\ chumank@jubilo.com \\ [Received May 26, 2011; Accepted November 25, 2011; Published online December 23, 2011]
}

\begin{abstract}
This study examined the relationships between longitudinal changes in data obtained in the YoYo intermittent recovery level 2 test (Yo-Yo IR2), maximal oxygen consumption ( $\dot{\mathbf{V}} \mathbf{O}_{2}$ max) and leg muscle size and function in pubescent soccer players. Forty-four soccer players with a mean age of $12.8 \pm 0.2$ years participated, and were classified into three maturational groups (Late, Average and Early) according to their peak height velocity age. The Yo-Yo IR2 and five-jump tests along with measurement of $\dot{\mathbf{V}} \mathbf{O}_{2}$ max and thigh muscle volume were conducted on two occasions 6 months apart. Performances in the Yo-Yo IR2 for the Late, Average and Early groups were $311 \pm 61,371 \pm 88$ and $411 \pm 72$ meters at the first measurement, and $389 \pm 73,509 \pm 117$ and $621 \pm 69$ meters 6 months later. Consequently, two-way repeated-measures ANOVA revealed a significant interaction whereby the improvement in Yo-Yo IR2 performance was larger in the Early group (51.3\%) than in the Late group $(\mathbf{2 4 . 8} \%)$. Greater increases of thigh muscle volume and the result of the five-jump test were found in the Early group, and these were correlated significantly $(r=0.52, p<0.05 ; r=0.39, p<0.05)$ with improvement of Yo-Yo IR2 performance. In contrast, there was no relationship $\left(r=-0.02\right.$, n.s.) between the increments of $\dot{\mathrm{V}} \mathrm{O}_{2}$ max and Yo-Yo IR2 performance. These results suggest that performance in the Yo-Yo IR2 is affected by maturity to a great extent, early-maturing players having an advantage in terms of leg muscle development.
\end{abstract}

Keywords: maturity status, peak height velocity, aerobic fitness, leg muscle power, growth and development

\section{Introduction}

Soccer is a physically demanding sport, and greater demands are placed on players at higher levels of competition (Mohr et al., 2003). A trend towards more physically demanding soccer over the last few decades has been documented in many scientific reports (Mohr et al., 2003; Reilly, 2005), leading to greater emphasis on the need for a higher level of fitness in order to succeed in the sport. For this reason, soccer training now includes physical exercises that improve fitness conditioning as well as technical or tactical skills, even in younger players.

Soccer is characterized by high-intensity intermittent activity involving a wide variety of fitness components (Bangsbo, 1994). High-intensity running among professional players has gradually increased over the last few decades (Mohr et al., 2003). To evaluate the fitness of soccer players for performing repeated high-intensity exercise, Bangsbo developed 
an intermittent exercise test called the "Yo-Yo intermittent recovery test" which requires the player to repeat a shuttle run of 20 meters at gradually increasing speed in each successive run (Bangsbo et al., 2008; Krustrup et al., 2003, 2006). There are two types of Yo-Yo intermittent recovery test: level 1 and level 2. According to Bangsbo et al. (2008) level 1 focuses on maximal activation of a player's aerobic energy system, whereas level 2 applies a heavy load to both the aerobic and anaerobic energy systems. It has been reported that the Yo-Yo intermittent recovery level 2 test (Yo-Yo IR2) can predict the distance covered during sprint and high-intensity running in professional soccer players (Randers et al., 2009) and that the results of Yo-Yo IR2 are better in players at higher levels of the sport (Krustrup et al., 2006; Randers et al., 2009). The Yo-Yo IR2 is also sensitive to fitness adaptation to training over the course of the season in professional soccer players (Krustrup et al., 2006). For these reasons, the Yo-Yo IR2 is utilized worldwide as one of the best practical fitness tests for high-level soccer players.

The structure and physiological validity of the YoYo intermittent recovery test has already been confirmed among not only adults but also young soccer players (Castagna et al., 2010). However, while the use of Yo-Yo IR2 has been well documented for adult soccer players, few Yo-Yo IR2 data are available for young soccer players. There is even less information on the data transitions necessary for identifying the relative contributions of various fitness components to Yo-Yo IR2 during growth. In adult players, Yo-Yo IR2 performance has been confirmed to correlate moderately with maximal oxygen consumption ( $\left.\dot{\mathrm{VO}}_{2} \max \right)$, causing Yo-Yo IR2 to be partly associated with maximal aerobic fitness (Bangsbo et al., 2008; Krustrup et al., 2006). In young soccer players aged 13 years however, we have reported that Yo-Yo IR2 performance showed no relation to $\dot{\mathrm{V}} \mathrm{O}_{2}$ max or anaerobic threshold related values (Chuman et al., 2009). We assume here that immaturity of the leg muscles of young soccer players limits their performance in the Yo-Yo IR2, even if they have high levels of aerobic fitness. The Yo-Yo IR2 consists of a shuttle run over a short distance that consequently imposes burdens of quick deceleration, changing direction, and re-acceleration, and so requires adequate leg muscle power. Improved Yo-Yo IR2 performance in young soccer players would therefore indicate either development of aerobic fitness, development of leg muscular factors, or both. However these relationships have not yet been clarified.

Spurts in $\dot{\mathrm{V}}_{2} \max$ and muscle power during adolescent development occur with different timings and tempos. Maximal development in $\dot{\mathrm{V}}_{2}$ max during growth is coincident with the age of peak height velocity (PHV), which is commonly used as a bench mark for maturation (Mirwald et al., 2002). However, the marked increase of muscle power in boys occurs slightly later than that of $\dot{\mathrm{VO}}_{2} \max$ (Beunen and Malina, 1988) and continues during late adolescence with an increased muscle volume caused by a rise in anabolic hormones relating to sexual maturation. The higher level of fitness in each component required in successful soccer players may be efficiently achieved by a synergy of natural growth and concomitant systemic training optimally designed for each developmental stage (Hansen et al., 1999). However, little information is available regarding development of the ability to repeatedly perform intense exercise in young soccer players.

In most cases, young soccer players practice and play the game along with groups of the same chronological age. However, even within the same single year of chronological age, there may be as much as five years difference in biological maturity status (Malina and Bouchard, 1991). According to Chuman et al. (2009), early-maturing soccer players who had already passed through their PHV period attained a Yo-Yo IR2 performance 32\% higher than that of their less mature counterparts, even at the same chronological age of 13 years. Yo-Yo IR2 data for young soccer players are therefore strongly affected by biological maturity, meaning that the results of the Yo-Yo IR2 should be interpreted with caution in this age group.

In the present study we evaluated the Yo-Yo IR2 results for pubescent soccer players relative to changes in other parameters determined 6 months apart. Our purpose was to clarify the relative contribution of aerobic and leg muscle-related factors to improved performance in the Yo-Yo IR2 in pubescent soccer players while taking their biological maturity into account. To this end, we divided subjects at almost the same chronological age from 12 to 13 years into three groups according to maturation, and related the increments in Yo-Yo IR2 results to development of aerobic and leg musclerelated factors. 


\section{Methods}

The subjects were 44 young male soccer players in a team managed by a club belonging to the Japan Professional Football League Division 1 (J1League). The subjects, with a mean age of $12.8 \pm 0.2$ years, were all in the U-13 (under the age of 13) category at the time of the first measurement (M1). The subjects' chronological and PHV ages were calculated to the first decimal place. All subjects were field players and had passed a selection process that judges the level of soccer skill required to join the team. Goalkeepers were not included in the study. The subjects had been engaged in supervised competitive soccer training for an average of $6.8 \pm 1.2$ years, with a minimum of 4 years. They attended the team training with written informed consent from their guardians, and this study was carried out as part of the training program under the direction of coaches with approval from the club's executives. The Yo-Yo IR2 and the other measurements were performed twice: in April immediately after team enrollment (M1: first measurement), and in October, six months after M1 (M2: second measurement). During those six months, each subject was coached under nearly the same weekly training schedule, which was 1.5 to 2 hours per day, 5 days per week and 1 game on the weekend. Physical training was conducted approximately twice per week, and was mainly long distance running, interval training, and circuit training using the player's own body mass as a load. Neither high-resistance training nor plyometric training was included. This study was approved by the Ethics Committee of the University of Tsukuba.

The biological maturity status of the subjects was classified according to their PHV age. The heights of the subjects from 6 to 15 years of age were obtained by reviewing school annual health reports, which had recorded the subjects' heights in April, September and January for 9 years. All height data were then applied to the BTT model (AUXAL Ver.3.0 for Windows, Scientific Software International) and their individual PHV ages were determined. The subjects were categorized into three maturational groups: Late, Average and Early. The Average group consisted of subjects whose chronological age was within \pm 0.5 years of their PHV age. The Late group consisted of subjects whose chronological age was 0.5 years prior to their PHV age, and the Early
Table 1 Chronological and PHV age of subjects

\begin{tabular}{lcccc}
\hline \multicolumn{1}{c}{ Item } & Total & Late & Average & Early \\
\hline Number of subjects & 44 & 14 & 15 & 15 \\
Chronological age (yrs) & $12.8 \pm 0.2$ & $12.8 \pm 0.2$ & $12.8 \pm 0.2$ & $12.9 \pm 0.1$ \\
PHV age (yrs) & $12.8 \pm 1.0$ & $14.0 \pm 0.7$ & $12.8 \pm 0.3$ & $11.8 \pm 0.5$ \\
\hline
\end{tabular}

group consisted of subjects whose chronological age had already exceeded their PHV age by 0.5 years. The subjects in the Late, Average and Early groups were therefore considered to be at maturational stages before, during and after their PHV period, respectively. The chronological ages at M1, PHV ages and the number of subjects in the Late, Average and Early groups are shown in Table 1.

Yo-Yo intermittent recovery level 2 test. The Yo-Yo IR2 is a field test whose reliability and validity have already been confirmed (Krustrup et al., 2006). The Yo-Yo IR2 in this study was carried out according to the manual, and included a $C D$ from the test provider (bangsbosport.com). The subjects repeated the 20-meter shuttle run starting at a signal from the $\mathrm{CD}$, turning at a second signal and returning to the starting point before a third and final signal. The interval at which the three signals sounded decreased with each subsequent shuttle run. As a rest, the subjects jogged for 10 seconds between runs, the jog requiring subjects to travel to a cone placed 5 meters away and return to the starting position. In the event a subject failed to return to the starting position prior to the third signal a total of two times, the measurement was concluded and the distances traveled were recorded as the Yo-Yo IR2 results.

The subjects performed the test on artificial turf while wearing soccer shoes. Before the test, the subjects warmed up for 15 minutes under the supervision of the coach by jogging, stretching, and some high-intensity running and jumping with sufficient rest. The test was conducted in the evening in the spring and fall, when the temperature was approximately $13-18^{\circ} \mathrm{C}$.

Maximal oxygen consumption. The subjects reported to the laboratory within 1 week after the Yo-Yo IR2 to measure their maximal oxygen consumption ( $\dot{\mathrm{V}}_{2} \mathrm{max}$ ) by an incremental test to exhaustion on a treadmill. The graded exercise test protocol was a 3minute submaximal run followed by a 1-minute rest, repeated 4 to 6 times. The submaximal running velocities were set to $180,200,220,240,260$ and 280 $\mathrm{m} \cdot \mathrm{min}^{-1}$. During this test, the heart rate and oxygen 
Table 2 Descriptive statistics of the three maturity groups

\begin{tabular}{|c|c|c|c|c|c|c|c|c|c|}
\hline \multirow{2}{*}{ Item } & \multicolumn{2}{|c|}{ Late $(\mathrm{N}=14)$} & \multicolumn{2}{|c|}{ Average $(\mathrm{N}=15)$} & \multicolumn{2}{|c|}{ Early $(\mathrm{N}=15)$} & \multicolumn{3}{|c|}{ ANOVA } \\
\hline & M1 & M2 & M1 & M2 & M1 & M2 & Maturity & Measurement & Interaction \\
\hline Height (m) & $1.51 \pm 0.06$ & $1.55 \pm 0.06$ & $1.57 \pm 0.05$ & $1.61 \pm 0.05$ & $1.66 \pm 0.05$ & $1.68 \pm 0.05$ & $* *$ & $* *$ & $* *$ \\
\hline Body mass (kg) & $39.4 \pm 5.1$ & $42.4 \pm 5.7$ & $44.4 \pm 3.7$ & $48.3 \pm 4.7$ & $54.7 \pm 7.6$ & $58.4 \pm 7.2$ & $* *$ & $* *$ & \\
\hline$\dot{\mathrm{V}} \mathrm{O}_{2} \max \left(\mathrm{L} \cdot \mathrm{min}^{-1}\right)$ & $2.51 \pm 0.29$ & $2.93 \pm 0.29$ & $3.01 \pm 0.26$ & $3.42 \pm 0.30$ & $3.54 \pm 0.39$ & $4.00 \pm 0.46$ & $* *$ & $* *$ & \\
\hline$\dot{\mathrm{V}} \mathrm{O}_{2} \max \left(\mathrm{mL} \cdot \mathrm{min}^{-1} \cdot \mathrm{kg}^{-1}\right)$ & $64.0 \pm 6.4$ & $69.6 \pm 5.7$ & $67.8 \pm 4.8$ & $70.9 \pm 4.5$ & $64.9 \pm 4.5$ & $68.7 \pm 4.1$ & & $* *$ & \\
\hline Thigh muscle volume (L) & $3.03 \pm 0.43$ & $3.44 \pm 0.51$ & $3.63 \pm 0.53$ & $4.12 \pm 0.54$ & $4.65 \pm 0.68$ & $5.18 \pm 0.64$ & ** & ** & * \\
\hline Five-jump test (m) & $9.55 \pm 0.61$ & $9.69 \pm 0.61$ & $10.07 \pm 0.63$ & $10.33 \pm 0.67$ & $10.58 \pm 0.41$ & $11.04 \pm 0.32$ & $* *$ & $* *$ & $*$ \\
\hline
\end{tabular}

uptake were monitored every 10 seconds with Polar Heart Rate Monitors (Polar Electro) and Meta Max (Cortex Biophysic GmbH). After submaximal running, a 2-minute rest was taken, and a final run to exhaustion was performed to determine the $\dot{\mathrm{V}} \mathrm{O}_{2}$ max. The running velocity for $\dot{\mathrm{V}}_{2}$ max determination was set to $280 \mathrm{~m} \cdot \mathrm{min}^{-1}\left(260 \mathrm{~m} \cdot \mathrm{min}^{-1}\right.$ in the case of subjects showing near-exhaustion at submaximal running below $240 \mathrm{~m} \cdot \mathrm{min}^{-1}$ ) but the inclination was increased by $1 \%$ every minute until volitional exhaustion after 3 minutes of maximal running. The $\dot{\mathrm{V}}_{2} \max$ was defined as the highest average value during 1 minute. Immediately after the final run, two blood samples were taken by finger prick for lactate analysis (Lactate Pro, Arkray Inc). In most cases, achievement of $\mathrm{V}_{2}$ max was confirmed by a plateau in the oxygen uptake increase of less than $80 \mathrm{~mL} \cdot \mathrm{min}^{-1}$ despite an increase in the inclination. In the other cases, achievement of $\dot{\mathrm{V}} \mathrm{O}_{2}$ max was considered as meeting the following criteria: 1) a respiratory exchange ratio of more than $1.10 ; 2$ ) a heart rate within \pm 10 beats $\cdot \mathrm{min}^{-1} 1$ of age-predicted maximal heart rate (220-age); 3) lactate concentration after the run of more than 8 $\mathrm{mmol} \cdot \mathrm{L}^{-1}$. The $\mathrm{VO}_{2}$ max is indicated as an absolute value $\left(\mathrm{L} \cdot \mathrm{min}^{-1}\right)$, and as a value relative to body mass $\left(\mathrm{mL} \cdot \mathrm{min}^{-1} \cdot \mathrm{kg}^{-1}\right)$.

Leg muscle power and volume. Leg muscle power was assessed by the five-jump test (Chamari et al., 2008), which consists of 5 step jumps starting from a standing position, alternating between legs, and completed on the fifth jump by landing on both legs. The shortest distance from the toe at the start, to the heel at landing after the fifth jump was measured and taken as the result of the five-jump test. Quantitative development of the thigh muscles on both sides was estimated by magnetic resonance imaging (Signa Profile 0.2-T scanner, General Electric Medical System). Each side of the thigh was scanned separately, using a scanning protocol similar to that employed in earlier studies (Hoshikawa et al., 2009; Masuda et al., 2003) In brief, longitudinal images of the thigh were obtained to identify the greater trochanter and lower end of the femur. A transverse T1 image with a thickness of $10 \mathrm{~mm}$ was then obtained 30\% (Lower), 50\% (Middle) and 70\% (Upper) along the length of the femur (TR350 ms, TE21 ms, matrix $256 \times 256$, FOV $40 \mathrm{~cm}, 2 \mathrm{NEX})$. In each of the obtained transverse images, the area of muscle, fat and femur were separated out using a computer mouse, and the muscle cross-sectional areas were calculated by multiplying the number of pixels in the muscle portion by the area per 1 pixel (1 pixel $=1.56 \mathrm{~mm}^{2}$ ). The thigh muscle volume was then calculated by multiplying the average of the muscle cross-sectional area of the three transverse images by the femur length. The thigh muscle volume averaged on both sides was used for analysis.

All measurement results are presented as the mean \pm standard deviation (Table 2). Two-way repeatedmeasures ANOVA ( 3 maturity groups $\times 2$ measurement times) was used to examine the differences in the improvement between M1 and M2 and among the groups. Whenever a significant interaction was observed, one-way ANOVA was used to locate significant differences in the increments among the groups. Simple linear regression analysis was used to calculate the coefficient of correlation between the changes in the Yo-Yo IR2 results and other measurement variables. SPSS 12.0J for Windows was used for all statistical analyses. The level of significance was set at $p<0.05$.

\section{Results}

Figure 1 shows the Yo-Yo IR2 results for all groups. The results for the Late, Average and Early groups were $311 \pm 61,371 \pm 88$ and $411 \pm 72 \mathrm{~m}$ at 
$\mathrm{M} 1$, and $389 \pm 73,509 \pm 117$ and $621 \pm 69 \mathrm{~m}$ at M2. The Yo-Yo IR2 results showed a significant interaction with both measurement time and maturity group $(F(2,41)=7.5, p<0.05)$. One-way ANOVA with post-hoc test indicated significant differences among the groups $(F(2.41)=7.5, p<0.05)$, and the improvement in Yo-Yo IR2 in the Early group

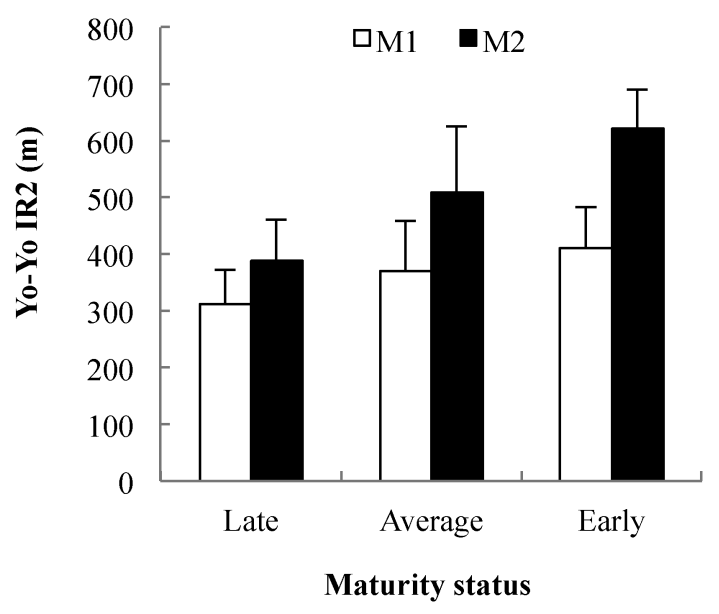

Figure 1 Yo-Yo IR2 performances at M1 and M2 Interaction was significant.
$(51.3 \%)$ was significantly larger than that in the Late group $(24.8 \%)$.

Absolute and relative $\dot{\mathrm{V}}_{2} \max$, thigh muscle volume and five-jump test results increased significantly during the 6 -month period $(F(1,41)=$ $184.6(p<0.01)$ for absolute $\dot{\mathrm{VO}}_{2} \max , F(1,41)=$ $45.2(p<0.01)$ for relative $\dot{\mathrm{VO}}_{2} \max , F(1,41)=$ $600.3(p<0.01)$ for thigh muscle volume, and $F(1$, $41)=33.1(p<0.01)$ for the five-jump test) (Table 2). All of them except for relative $\dot{\mathrm{VO}}_{2}$ max also showed significant differences among the maturity groups $(F(2,41)=38.2(p<0.01)$ for absolute $\dot{\mathrm{V}} \mathrm{O}_{2} \max , F(2,41)=1.7$ (n.s.) for relative $\dot{\mathrm{V}}_{2} \max$, $F(2,41)=33.7(p<0.01)$ for thigh muscle volume, $F(2,41)=18.5(p<0.01)$ for five-jump test) (Table 2). No interaction was found for $\dot{\mathrm{V}}_{2}$ max expressed as either an absolute $(F(2,41)=0.3$, n.s. $)$ or relative value $(F(2,41)=1.2$, n.s. $)$, indicating that the increase during the 6-month period did not differ between the groups. Significant interactions were found in thigh muscle volume $(F(2,41)=3.4$, $p<0.05)$ and the results of the five-jump test $(F(2$, $41)=3.6, p<0.05)$, the Early group showing significantly larger improvements than the Late group

Table 3 The correlation coefficients between increment of Yo-Yo IR2 result and changes of the other variables

\begin{tabular}{lcccc}
\hline \multirow{2}{*}{ Item (Change) } & \multicolumn{3}{c}{ Increment of Yo-Yo IR2 result $(\mathrm{m})$} \\
\cline { 2 - 5 } & Total $(\mathrm{N}=44)$ & Late $(\mathrm{N}=14)$ & Average $(\mathrm{N}=15)$ & Early $(\mathrm{N}=15)$ \\
\hline Height $(\mathrm{m})$ & -0.10 & 0.22 & 0.09 & 0.25 \\
Body mass $(\mathrm{kg})$ & 0.07 & 0.07 & -0.26 & 0.31 \\
$\dot{\mathrm{V} O}{ }_{2} \max \left(\mathrm{L} \cdot \mathrm{min}^{-1}\right)$ & 0.09 & 0.46 & -0.24 & 0.29 \\
$\dot{\mathrm{VO}}$ max $\left(\mathrm{mL} \cdot \mathrm{min}^{-1} \cdot \mathrm{kg}^{-1}\right)$ & -0.02 & 0.49 & -0.15 & 0.14 \\
Thigh muscle volume (L) & $\mathbf{0 . 5 2}^{*}$ & 0.20 & 0.18 & $\mathbf{0 . 7 6}^{*}$ \\
Five-jump test (m) & $\mathbf{0 . 3 9}^{*}$ & 0.24 & 0.10 & $\mathbf{0 . 5 5}^{*}$ \\
\hline
\end{tabular}

$* ; p<0.05$
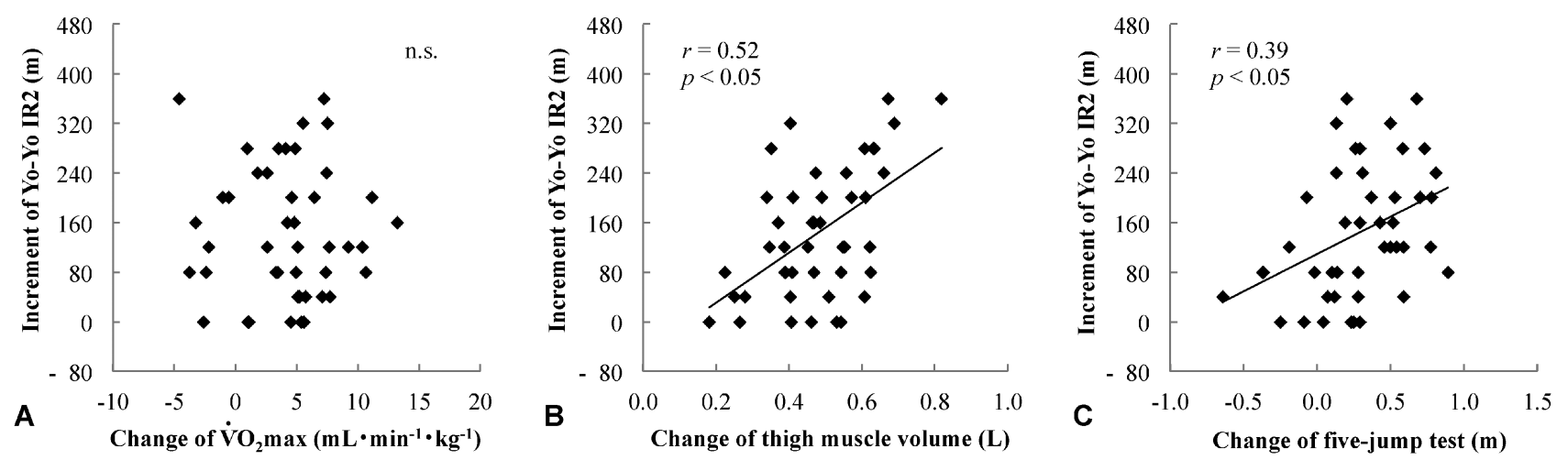

Figure 2 The relationship between increment of Yo-Yo IR2 performance and change of $\dot{\mathrm{V}}_{2} \mathrm{max}(\mathbf{A})$, thigh muscle volume (B) and five-jump test (C) 
(Table 2).

Table 3 shows the results of analysis of correlations between increments in the Yo-Yo IR2 and other variables. The changes in $\mathrm{V}_{2}$ max did not correlate significantly with increased Yo-Yo IR2 performance, even when pooled data for all groups were considered (Figure 2A). However, improvements in thigh muscle volume and the results of the five-jump test both showed significant relationships with the increase in Yo-Yo IR2 performance, with respective correlation coefficients of 0.52 and 0.39 (Figure 2B, 2C). Moreover, the correlation coefficients were much higher for the Early group, at 0.76 and 0.55 , respectively (Table 3). However, there were no significant correlations with the increase in Yo-Yo IR2 performance among any of the variables in the Late and Average groups.

\section{Discussion}

In a previous pilot study, we found that the test performance of U-13 soccer players in the Yo-Yo IR2 was strongly affected by maturation stage (Chuman et al., 2009). In the present study, we evaluated changes in the Yo-Yo IR2 test results during a 6month period in $\mathrm{U}-13$ players. We also investigated the relationship between changes in $\dot{\mathrm{V}}_{2}$ max and development of leg muscle size and function while taking the subject's maturity status into account. During the 6-month period, the players experienced almost the same volume of training. Nevertheless, a significant interaction between measurement time and maturity was observed in terms of Yo-Yo IR2 performance (Figure 1); the improvement in the Early group was more than twice that in the Late group, thus confirming the positive effect of maturation on Yo-Yo IR2 performance in pubescent soccer players.

Changes in $\dot{\mathrm{V}}_{2}$ max were not related to improved Yo-Yo IR2 performance in the present study subjects (Figure 2). Yo-Yo IR2 performance reflects $\dot{\mathrm{VO}}_{2}$ max in adult soccer players (Bangsbo et al., 2008; Krustrup et al., 2006). In the present study, all the groups showed similar significant increments in $\dot{\mathrm{V}} \mathrm{O}_{2}$ max, irrespective of whether it was expressed as an absolute value $(14.2 \%)$ or relative to body mass $(6.3 \%)$, in spite of a significant gain of $3.5 \mathrm{~kg}$ in body mass (Table 2). This result indicates that the subjects developed maximal aerobic fitness by training, or through synergy between training and growth, during a 6-month period of natural maturation. Considering the high basic level of aerobic fitness in our subjects (average $\mathrm{VO}_{2} \max =65.6 \mathrm{~mL}$. $\min ^{-1} \cdot \mathrm{kg}^{-1}$ ), the degree of change observed in $\mathrm{V}_{2}$ max was not small, and was comparable to the improvements reported by Helgerud et al. (2001), who found that this degree of increment in aerobic fitness induced improvements in soccer performance relative to the distance covered, number of sprints, number of involvements with the ball, and average work intensity during a soccer game. In general, a higher $\dot{\mathrm{V}}_{2}$ max is assumed to assist faster recovery during high-intensity intermittent exercise (Balsom et al., 1992; Reilly, 1997). However, our results showed that this remarkable improvement in $\dot{\mathrm{V}} \mathrm{O}_{2} \max$ was unrelated to improvements in Yo-Yo IR2, suggesting that it is not crucial for pubescent soccer players and has little effect in determining YoYo IR2 performance.

In contrast, the change in thigh muscle volume was moderately correlated with improved Yo-Yo IR2 performance, with a correlation coefficient of 0.52 , and similar results were obtained for the fivejump test performance, which was also significantly correlated $(r=0.39)$ with improved Yo-Yo IR2 performance (Figure 2). The calculated average running velocity in the second and third runs of Yo-Yo IR2 was 250 and $267 \mathrm{~m} \cdot \mathrm{min}^{-1}$, respectively, being faster on average than the running velocity at OBLA $\left(245 \mathrm{~m} \cdot \mathrm{min}^{-1}\right)$ observed previously in pubescent soccer players aged 12-13 years (Chuman et al., 2009). Therefore, after the second or third Yo-Yo IR2 run, the subjects were made to exert leg muscle power to accelerate, decelerate and turn quickly within the limited available time. Since thigh muscle volume and five-jump test performance were correlated significantly in this study $(r=0.70$ and 0.60 at M1 and M2), greater leg muscle size and function were consequently found to lead to better Yo-Yo IR2 performance in pubescent soccer players. Therefore, it is thought that rather than aerobic fitness, quantitative and qualitative development of leg muscle over the 6-month study period played a significant role in improving Yo-Yo IR2 performance.

However, the coefficients of correlation between the improvement in Yo-Yo IR2 performance and the changes in both thigh muscle volume and five-jump test performance were by no means high. When the subjects were classified into three maturational groups, the changes in thigh muscle volume and five- 
jump test performance in the Early group showed a stronger correlation with improved Yo-Yo IR2 performance, with correlation coefficients of 0.76 and 0.55, respectively (Table 3). However, the changes in thigh muscle volume and five-jump test performance in the other two groups were unrelated to improved Yo-Yo IR2 performance (Table 3). The adolescent growth spurt associated with changing levels of anabolic growth hormones and muscle volume gain at this time is a direct source of remarkable development of muscle power in pubescent males (Malina and Bouchard, 1991). Philippaerts et al. (2006) reported that peak muscle power gain occurred at, and continued after PHV age in youth soccer players. In line with this, we also confirmed that even though both groups experienced the same training regime during the 6-month study period, the Early group showed a tendency for greater development of thigh muscle volume and improvement in five-jump test performance (Figure 2) compared with the Late group. This tendency contrasted with the observed changes in $\mathrm{V}_{2}$ max, which did not differ among the groups. Taking these results as a whole, it is reasonable to assume that leg musclerelated factors are major determinants of improvement in Yo-Yo IR2 performance, at least in young soccer players just after PHV age, when there is considerable development of leg muscle size and function.

Bangsbo et al. (2008) pointed out that performance in the Yo-Yo intermittent recovery level 1 test for junior soccer players progressed linearly with a corresponding increase in age from 12 to 18 years, and that the Yo-Yo IR2 test performance of players aged 16 to 17 years was still as much as $30 \%$ lower than that for older elite soccer players. This tendency also appears likely in the case of Japanese topelite players. The Japan Football Association (2005) reported that whereas Yo-Yo IR2 performance in the Japan national team was $1019 \mathrm{~m}$, performance in the U-18, U-15 and U-13 national teams was 935 $\mathrm{m}, 781 \mathrm{~m}$, and $633 \mathrm{~m}$, respectively. Among high-level soccer players, juniors aged 16 to 17 years had already achieved an aerobic capacity comparable to that of professionals (Bangsbo, 1994; McMillan et al., 2005). On the other hand, large differences in thigh muscle cross-sectional area and knee strength were reported between juniors at late adolescence and seniors, even among high-level players (Hoshikawa et al., 2009). The age-related boost of
Yo-Yo intermittent recovery test performance observed among high-level soccer players may therefore indicate that the ability to perform repeated intense exercise is strongly affected by leg muscle-related factors. Conversely, the fact that $\dot{\mathrm{VO}}_{2}$ max correlates moderately with Yo-Yo IR2 in adult soccer players (Krustrup et al., 2006) raises the question of from what age the $\dot{\mathrm{VO}}_{2}$ max is effective in improving Yo-Yo IR2 results or the ability to perform repeated high-intensity exercise. We have reported that $\dot{\mathrm{VO}}_{2} \max$ is correlated with Yo-Yo IR2 performance in players aged 16 to 17 years, but not in players aged 12 to 13 years (Chuman et al., 2011). Therefore we assume that aerobic and muscle-related factors contribute to changes in Yo-Yo IR2 performance through puberty to late adolescence. However, this should be investigated in greater detail in a future study.

One fact emerging from this study is that caution should be used when interpreting Yo-Yo IR2 results among young players around the time of puberty. Moreover, maturational stage affected Yo-Yo IR2 performance to a great extent in terms of the development of leg muscle size and function, making it difficult to evaluate improvements of aerobic performance based on Yo-Yo IR2 results during the 6month training period in this subject cohort. Earlymaturing players after PHV age were found to exhibit a greater improvement in Yo-Yo IR2 performance than their later-maturing counterparts before PHV age because of the more advanced development of leg muscle size and function, irrespective of aerobic fitness during the 6 months of training.

\section{Conclusion}

Maturity status affected improvement of Yo-Yo IR2 performance over a 6-month period in junior soccer players aged 12 to 13 years. The incremental increase of Yo-Yo IR2 performance in early-maturing players after $\mathrm{PHV}$ age was more than twice that in their later-maturing counterparts before PHV age. The difference due to maturation stage is possibly attributable to differences in development of leg muscle size and function rather than aerobic fitness.

\section{References}

Balsom, P. D., Seger, J. Y., Sjödin, B., and Ekblom, B. (1992). Maximal-intensity intermittent exercise: effect of recovery duration. Int. J. Sports Med., 13(7): 528-533. 
Bangsbo, J. (1994). The physiology of soccer-with special reference to intense intermittent exercise. Acta Physiol. Scand., 151 (Suppl. 619): 1-156.

Bangsbo, J., Iaia, F. M., and Krustrup, P. (2008). The Yo-Yo intermittent recovery test: a useful tool for evaluation of physical performance in intermittent sports. Sports Med., 38(1): 37-51.

Beunen, G., and Malina, R. M. (1988). Growth and physical performance relative to the timing of the adolescent spurt. Exerc. Sport Sci. Rev., 16: 503-540.

Castagna, C., Manzi, V., Impellizzeri, F., Weston, M., and Barbero Alvarez, J. C. (2010). Relationship between endurance field tests and match performance in young soccer players. J. Strength Cond. Res., 24(12): 3227-3233.

Chamari, K., Chaouachi, A., Hambli, M., Kaouech, F., Wisloff, U., and Castagna, C. (2008). The five-jump test for distance as a field test to assess lower limb explosive power in soccer players. J. Strength Cond. Res., 22(3): 944-950.

Chuman, K., Hoshikawa, Y., and Iida, T. (2009). Yo-Yo intermittent recovery level 2 test in pubescent soccer players with relation to maturity category. Football Science 6: 1-6. (http: //www.shobix.co.jp/jssf/tempfiles/journal/2009/025.pdf)

Chuman, K., Hoshikawa, Y., Iida, T., and Nishijima, T. (2011). Relationships between Yo-Yo intermittent recovery tests and development of aerobic and anaerobic fitness in U-13 and U-17 soccer players. Int. J. Sport Health Sci., 9: 9197. (http://www.jstage.jst.go.jp/article/ijshs/9/0/91/_pdf/ -char/ja)

Hansen, L., Bangsbo, J., Twisk, J., and Klausen, K. (1999). Development of muscle strength in relation to training level and testosterone in young male soccer players. J. Appl. Physiol., 87(3): 1141-1147.

Helgerud, J., Engen, L. C., Wisloff, U., and Hoff, J. (2001). Aerobic endurance training improves soccer performance. Med. Sci. Sports Exer., 33(11): 1925-1931.

Hoshikawa, Y., Iida, T., Muramatsu, M., Nakajima, Y., Fukunaga, T., and Kanehisa, H. (2009). Differences in thigh muscularity and dynamic torque between junior and senior soccer players. J. Sports Sci., 27(2): 129-138.

Japan Football Association (2005). Evaluation of physical performance 2006. Tokyo: Japan Football Association (in Japanese).

Krustrup, P., Mohr, M., Amstrup, T., Rysgaard, T., Johansen, J., Steensberg, A., Pedersen, P. K., and Bangsbo, J. (2003). The Yo-Yo intermittent recovery test: physiological response, reliability, and validity. Med. Sci. Sports Exer., 35(4): 697-705.

Krustrup, P., Mohr, M., Nybo, L., Jensen, J. M., Nielsen, J. J., and Bangsbo, J. (2006). The Yo-Yo IR2 test: physiological response, reliability, and application to elite soccer. Med. Sci. Sports Exer., 38(9): 1666-1673.

Malina, R. M., and Bouchard, C. (1991). Growth, maturation and physical activity. Champaign: Human Kinetics.

Masuda, K., Kikuhara, N., Takahashi, H., and Yamanaka, K. (2003). The relationship between muscle cross-sectional area and strength in various isokinetic movements among soccer players. J. Sports Sci., 21: 851-858.

McMillan, K., Helgerud, J., Macdonald, R., and Hoff, J. (2005). Physiological adaptations to soccer specific endurance training in professional youth soccer players. Br. J. Sports Med., 39(5): 273-277.

Mirwald, R. L., Baxter-Jones, A. D. G., Bailey, D. A., and Beunen, G. P. (2002). An assessment of maturity from an- thropometric measurements. Med. Sci. Sports Exer., 34(4): 689-694.

Mohr, M., Krustrup, P., and Bangsbo, J. (2003). Match performance of high-standard soccer players with special reference to development of fatigue. J. Sports Sci. 21(7): 519-528.

Philippaerts, R. M., Vaeyens, R., Janssens, M., Renterghem, B. V., Matthys, D., Craen, R., Bourgois, J., Vrijens, J., Beunen, G., and Malina, R. M. (2006). The relationship between peak height velocity and physical performance in youth soccer players. J. Sports Sci., 24(3): 221-230.

Randers, M. B., Jensen, J. M., Bangsbo, J., and Krustrup, P. (2009). Match performance and Yo-Yo IR2 test performance of players from successful and unsuccessful professional soccer teams. In T. Reilly \& F. Korkusuz (eds.), Science and Football VI. (pp. 345-349). New York: Routledge.

Reilly, T. (2005). An ergonomics model of the soccer training process. J. Sports Sci., 23(6): 561-572.

Reilly, T. (1997). Energetics of high-intensity exercise (soccer) with particular reference to fatigue. J. Sports Sci., 15(3): 257-263.

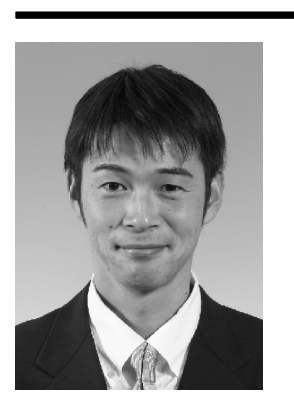

Name:

Kentaro Chuman

\begin{abstract}
Affiliation:
Doctoral Program in Physical Education, Health and Sport Sciences, University of Tsukuba YAMAHA FOOTBALL CLUB CO., LTD.
\end{abstract}

\section{Address:}

2500 Shingai, Iwata-shi, Shizuoka 438-0025 Japan

Brief Biographical History:

2003-2005 Master's Program in Health and Physical Education, University of Tsukuba

2009-2011 Doctoral Program in Physical Education, Health and Sport Sciences, University of Tsukuba

2004-2011 YAMAHA FOOTBALL CLUB CO., LTD.

Main Works:

-Chuman, K., Takahashi, S. and Nishijima, T. (2004). Dynamic characteristics of muscle in preadolescent boys. Human Performance Measurement 1: 30-35.

- Chuman, K., Takahashi, S., Nakano, T. and Nishijima, T. (2005). Dynamic causal structure analysis of condition fluctuation factors in a soccer player. Football Science 2: 1-7.

- Chuman, K., Hoshikawa, Y. and Iida, T. (2009). Yo-Yo intermittent recovery level 2 test in pubescent soccer players with relation to maturity category. Football Science 6: 1-6.

-Chuman, K., Hoshikawa, Y., Iida, T. and Nishijima, T. (2011). Relationships between Yo-Yo intermittent recovery tests and development of aerobic and anaerobic fitness in U-13 and U-17 soccer players. International Journal of Sport and Health Science 9: 91-97.

Membership in Learned Societies:

Japanese Society of Science and Football 